\title{
Direct Detection of Mycobacterium tuberculosis Complex in Nonrespiratory Specimens by Gen-Probe Amplified Mycobacterium Tuberculosis Direct Test
}

\author{
FREDY GAMBOA,${ }^{1,2}$ JOSÉ M. MANTEROLA, ${ }^{1,2}$ BELÉN VINADO,${ }^{1}$ LOURDES MATAS, ${ }^{1,2}$ \\ MONTSERRAT GIMÉNEZ, ${ }^{1}$ JOAN LONCA, ${ }^{1}$ JOAN RUIZ MANZANO,${ }^{3}$ CARLOS RODRIGO, ${ }^{4}$

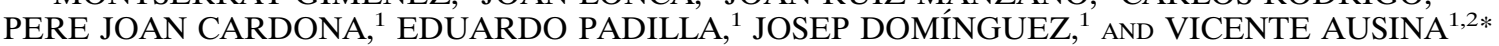 \\ Servicios de Microbiología, ${ }^{1}$ Neumología, ${ }^{3}$ and Pediatría, ${ }^{4}$ Hospital Universitario Germans Trias $i$ Pujol, \\ and Departamento de Genética y Microbiología, Facultad de Medicina, \\ Universidad Autónoma de Barcelona, ${ }^{2}$ Barcelona, Spain
}

Received 3 July 1996/Returned for modification 15 August 1996/Accepted 18 October 1996

\begin{abstract}
The Gen-Probe Amplified Mycobacterium Tuberculosis Direct Test (AMTDT) was adapted for the detection of Mycobacterium tuberculosis complex in 224 nonrespiratory specimens from 188 patients. The sensitivity and specificity of the AMTDT for such specimens, after resolution of discrepant results, were 85.7 and $100 \%$, respectively. Pretreatment of nonrespiratory specimens with sodium dodecyl (lauryl) sulfate is mandatory to obtain consistent and reproducible AMTDT results. The use of $500 \mu \mathrm{l}$ of decontaminated specimen improves the sensitivity of the test. Because the AMTDT detects stable rRNA from noncultivable bacilli, it is not useful for monitoring patients receiving treatment.
\end{abstract}

The laboratory diagnosis of tuberculosis (TB) is currently based on acid-fast staining and culture on solid and/or liquid media. Staining is a rapid screening test, but its sensitivity is low, especially in nonrespiratory specimens (7). Culture on solid media requires up to 6 weeks to detect positive specimens $(1,10)$. The faster BACTEC radiometric broth system has been an important addition to culturing methods $(2,10,16)$. The use of high-performance liquid chromatography for the assay of mycolic acids (6) and the use of DNA probes $(14,27)$ have further aided in the rapid identification of Mycobacterium tuberculosis complex in culture.

In research laboratories, PCR has proven useful for the direct diagnosis of TB by allowing use of diverse genetic elements as the target templates $(1,5,8,10,11,23)$. Using noncommercial PCR assays, however, results in an unexpectedly high variation in sensitivity $(8,15,19,23)$. Another practical problem of PCR is that it does not fit easily into routine laboratory practice, as costly equipment and expertise are not always available $(8,11,19,23)$. Therefore, the search for rapid, standardized, and reliable commercial detection systems for $M$. tuberculosis continues $(5,17)$.

The Amplified Mycobacterium Tuberculosis Direct Test (AMTDT) (Gen-Probe Inc., San Diego, Calif.) is based on the isothermal amplification of target rRNA by DNA intermediates followed by chemiluminiscent detection of amplicons with an acridinium ester-labeled DNA probe. The AMTDT is highly sensitive and specific for detecting $M$. tuberculosis in respiratory specimens $(3,12,18,20,22,26)$. One drawback to using this test in the daily laboratory routine is that the manufacturers do not recommend its use with nonrespiratory specimens.

The purpose of the present study was threefold: (i) to evaluate and adapt the AMTDT for the detection of M. tuberculosis complex in nonrespiratory samples and compare it with standard culture and staining techniques, (ii) to investigate

\footnotetext{
* Corresponding author. Mailing address: Servicio de Microbiología, Hospital Universitario Germans Trias i Pujol, Carretera de Canyet s/n, 08916 Badalona, Spain. Phone: 39530 61. Fax: 4653924.
}

whether using 500 instead of $50 \mu$ l of sample improves AMTDT performance, and (iii) to study the viability of the AMTDT for the follow-up of patients receiving anti-TB treatment.

From February through December 1995, we investigated 224 consecutive nonrespiratory specimens (40 urine, 38 fecal, 28 biopsy or lymph node exudate, 1 pericardic fluid, 41 pleural exudate, 17 cerebrospinal fluid [CSF], 10 articular fluid, 17 ascitic fluid, 2 gastric juice aspirate, 13 liver or skin biopsy, 1 otic exudate, and 16 bone marrow aspirate samples) collected from 188 patients at our hospital. Upon receipt, specimens were stored at $4{ }^{\circ} \mathrm{C}$ prior to processing. Gastric aspirates were immediately neutralized with trisodium phosphate buffer after retrieval. Tissue specimens were sliced and homogenized in a mortar under sterile conditions before processing. Urine and other fluid samples were previously centrifuged at $3,600 \times g$ for $20 \mathrm{~min}$. These samples were digested and decontaminated with sodium dodecyl (lauryl) sulfate (SDS)-NaOH as previously described (28), the sediment obtained was washed with $30 \mathrm{ml}$ of distilled water and centrifuged $(3,300 \times g, 20 \mathrm{~min})$, and the supernatant was removed. Bone marrow aspirates were received in Isolator tubes (Wampole Laboratories, Cranbury, N.J.), and $1 \mathrm{ml}$ of sample was treated with $100 \mu \mathrm{l}$ of $10 \%$ SDS. After being vortexed for $5 \mathrm{~min}$, the samples were rinsed with 30 $\mathrm{ml}$ of distilled water and centrifuged $(3,300 \times \mathrm{g}, 20 \mathrm{~min})$, and the supernatant was removed. If the sediment still had hemorrhagic contents, we repeated the process. For all specimens, half the sediment was stored at $-80^{\circ} \mathrm{C}$ for the target-amplified test, and the other half was inoculated onto the culture media and used for acid-fast staining.

The smears were stained with auramine-rhodamine fluorochrome as a screening method (16). Positive slides were confirmed to be positive by the Ziehl-Neelsen stain. Clinical specimens were inoculated onto a Löwenstein-Jensen slant, onto a Coletsos slant (9), and into BACTEC 12B medium. Slants and vials were incubated at 35 to $37^{\circ} \mathrm{C}$ for up to 8 weeks. Solid media were read weekly, and BACTEC cultures were read twice weekly for the first 2 weeks and once weekly thereafter. Routine biochemical methods (16), gas-liquid chromatography 
(21), and Accuprobe culture confirmation tests (Gen-Probe Inc.) were employed for the identification of isolates.

The AMTDT was performed with $50 \mu l$ of pretreated (SDS$\mathrm{NaOH}$ ) specimen according to the manufacturer's package insert. Each run included positive and negative amplification controls and positive and negative hybridization controls. A positive cutoff of 30,000 relative light units was used. All specimens with discrepant results were retested with $50 \mu$ l of the same decontaminated sample. Additionally, false-negative samples were reevaluated by AMTDT using $500 \mu$ of the pellet as previously described (4). Briefly, $500 \mu \mathrm{l}$ of decontaminated sample was centrifuged $(5 \mathrm{~min}$ at $11,000 \times g$ in an Eppendorf Microfuge), and $50 \mu$ l of the pellet was resuspended in $200 \mu \mathrm{l}$ of specimen dilution buffer and processed according to the manufacturer's instructions. Cultures were the standards against which AMTDT results were compared.

In the cases where results from the culture and the AMTDT were discrepant, clinical data and histological and other microbiological results of the patient were evaluated. The sensitivity, specificity, positive predictive value (PPV), and negative predictive value (NPV) of the AMTDT were calculated in comparison with culture results and, separately, in comparison with culture results plus the patients' clinical data. Statistical comparisons were performed by using a chi-square test.

Two hundred twenty-four specimens from 188 patients with clinical signs or symptoms of extrapulmonary TB were tested. The sources of these specimens and detailed results of smears for acid-fast bacilli, cultures, and AMTDTs are given in Table 1. Seventy-one specimens from 60 patients (13 smear positive) were AMTDT positive and culture positive for $M$. tuberculosis, and 121 specimens (all smear negative and culture negative) from 100 patients were AMTDT negative. Twelve specimens (eight smear positive) were AMTDT negative and culture positive for nontuberculous mycobacteria (NTM); the species of NTM identified from these specimens were $M$. avium-M. intracellulare complex (four specimens), M. genavense (three specimens), M. xenopi (one specimen), M. kansasii (one specimen), and $M$. gordonae (three specimens). All NTM isolates from smear-positive specimens corresponded to patients with clinical pictures compatible with extrapulmonary or disseminated mycobacteriosis.

Results of 20 nonrespiratory specimens from 16 patients were discrepant (Table 2). Thirteen specimens (12 smear negative) were AMTDT negative and culture positive for $M$. tuberculosis. Twelve of these specimens gave cultures with $\leq 10$ colonies of $M$. tuberculosis, and one gave $\leq 100$ colonies; these 13 specimens were considered false negatives. Six AMTDTpositive and culture-negative samples corresponded to patients with active TB who were receiving anti-TB treatment at the moment they were enrolled in the study. Five samples corresponded to patients with other previous or intercurrent positive cultures for $M$. tuberculosis. These six specimens were considered true positives. One AMTDT-positive but culturenegative lymph node specimen remained inconclusive (Table 2 ). Of the 76 patients with extrapulmonary TB, 44 also had pulmonary disease.

The sensitivity, specificity, PPV, and NPV of the AMTDT for nonrespiratory specimens were 85.7, 100, 100, and 91.1\%, respectively. For smear-positive and smear-negative specimens culture positive for M. tuberculosis the sensitivities of AMTDT were 95 and $83 \%$, respectively. Comparisons of culture and AMTDT results for different types of extrapulmonary specimens are given in Table 3.

Discrepant results were retested with a new aliquot $(50 \mu \mathrm{l})$ of the same processed specimen. After repeat testing, results for seven AMTDT-positive and culture-negative specimens
TABLE 1. Comparison of results obtained in 224 nonrespiratory specimens by microscopy, culture, and Gen-Probe AMTDT

\begin{tabular}{|c|c|c|c|c|c|}
\hline \multirow{2}{*}{$\begin{array}{l}\text { Type of } \\
\text { specimen }\end{array}$} & \multirow[b]{2}{*}{$n$} & \multirow{2}{*}{$\begin{array}{l}\text { Microscopy } \\
\text { result }\end{array}$} & \multicolumn{2}{|r|}{ Culture } & \multirow{2}{*}{$\begin{array}{l}\text { AMTD' } \\
\text { result }\end{array}$} \\
\hline & & & Result & $\begin{array}{c}\text { Species } \\
\text { identified }\end{array}$ & \\
\hline Urine & 3 & Pos. ${ }^{b}$ & Pos. & M. tuberculosis & Pos. \\
\hline Urine & 6 & Neg. ${ }^{c}$ & Pos. & M. tuberculosis & Pos. \\
\hline Urine & 6 & Neg. & Pos. & M. tuberculosis & Neg. \\
\hline Urine & 3 & Neg. & Pos. & M. gordonae & Neg. \\
\hline Urine & 22 & Neg. & Neg. & & Neg. \\
\hline Fecal & 4 & Pos. & Pos. & M. tuberculosis & Pos. \\
\hline Fecal & 3 & Pos. & Pos. & MAIC $^{a}$ & Neg. \\
\hline Fecal & 1 & Pos. & Pos. & M. tuberculosis & Neg. \\
\hline Fecal & 3 & Pos. & Neg. & M. genavense & Neg. \\
\hline Fecal & 1 & Pos. & Pos. & M. xenopi & Neg. \\
\hline Fecal & 1 & Pos. & Neg. & & Pos. \\
\hline Fecal & 2 & Neg. & Pos. & M. tuberculosis & Pos. \\
\hline Fecal & 1 & Neg. & Pos. & MAIC & Neg. \\
\hline Fecal & 22 & Neg. & Neg. & & Neg. \\
\hline Lymph node & 5 & Pos. & Pos. & M. tuberculosis & Pos. \\
\hline Lymph n & 3 & Pos. & Neg. & & Pos. \\
\hline Lymph node & 9 & Neg. & Pos. & M. tuberculosis & Pos. \\
\hline Lymph node & 3 & Neg. & Pos. & M. tuberculosis & Neg. \\
\hline Lymph & 1 & Neg. & Neg. & & Pos. \\
\hline Lymph n & 7 & Neg. & Neg. & & Neg. \\
\hline Pericardic fluid & 1 & Neg. & Pos. & M. tuberculosis & Pos. \\
\hline Pleural exudate & 1 & Pos. & Pos. & M. tuberculosis & Pos. \\
\hline Pleural e & 1 & Pos. & Pos. & M. kansasii & Neg. \\
\hline Pleural e & 12 & Neg. & Pos. & M. tuberculosis & Pos. \\
\hline Pleural exudate & 27 & Neg. & Neg. & & Neg. \\
\hline CSF & 5 & Neg. & Pos. & M. tuberculosis & Pos. \\
\hline CSF & 3 & Neg. & Pos. & M. tuberculosis & Neg. \\
\hline CSF & 9 & Neg. & Neg. & & Neg. \\
\hline Articular fluid & 5 & Neg. & Pos. & M. tuberculosis & Pos. \\
\hline Articular fluid & 5 & Neg. & Neg. & & Neg. \\
\hline Ascitic fluid & 11 & Neg. & Pos. & M. tuberculosis & Pos. \\
\hline Ascitic fluid & 6 & Neg. & Neg. & & Neg. \\
\hline Gastric aspirate & 1 & Neg. & Pos. & M. tuberculosis & Pos. \\
\hline Gastric a & 1 & Neg. & Neg. & & Neg. \\
\hline Tissue biopsy & 2 & Pos. & Neg. & & Pos. \\
\hline Tissue biopsy & 1 & Neg. & Pos. & M. tuberculosis & Pos. \\
\hline Tissue biopsy & 10 & Neg. & Neg. & & Neg. \\
\hline Bone marrow & 4 & Neg. & Pos. & M. tuberculosis & Pos. \\
\hline & 12 & Neg. & Neg. & & Neg. \\
\hline Otic exudate & 1 & Neg. & Pos. & M. tuberculosis & Pos. \\
\hline
\end{tabular}

${ }^{a}$ MAIC, M. avium-M. intracellulare complex.

${ }^{b}$ Pos., positive.

${ }^{c}$ Neg., negative.

were confirmed; six were considered true positives, while one remained inconclusive. Thirteen AMTDT-negative and culture-positive specimens for M. tuberculosis were also confirmed as true negatives after repeating the AMTDT. However, 9 of these 13 specimens were AMTDT positive when $500 \mu l$ of sediment was used (Table 2). Overall, the sensitivity of the modified AMTDT using $500 \mu$ l of sediment was $95 \%(P<$ 0.001).

Twelve specimens from patients treated for active TB were selected for inclusion in the study. Six were AMTDT positive and culture positive for M. tuberculosis, and all were obtained from five human immunodeficiency virus-positive patients with multidrug-resistant TB. The other six specimens, from six patients treated for active TB for 1 to 7 months, were AMTDT positive, smear positive, and culture negative.

The AMTDT has recently been evaluated in comparison to culture methods and acid-fast bacillus smear for respiratory specimens $(1,3,18,20,25)$. For more than 5,000 respiratory 
TABLE 2. Analysis of discrepant results in nonrespiratory specimens ${ }^{a}$

\begin{tabular}{|c|c|c|c|c|c|c|c|c|}
\hline \multirow{3}{*}{$\begin{array}{l}\text { Type of } \\
\text { specimen }\end{array}$} & \multirow{3}{*}{$n$} & \multicolumn{4}{|c|}{ Result of: } & \multirow{3}{*}{$\begin{array}{l}\text { Antituberculosis } \\
\text { treatment (duration) }\end{array}$} & \multirow{3}{*}{$\begin{array}{l}\text { M. tuberculosis isolate } \\
\text { in other specimens }\end{array}$} & \multirow{3}{*}{ Final result } \\
\hline & & \multirow{2}{*}{ Microscopy } & \multirow{2}{*}{ Culture $^{b}$} & \multicolumn{2}{|c|}{ AMTDT with: } & & & \\
\hline & & & & $50 \mu \mathrm{l}$ & $500 \mu \mathrm{l}^{\mathrm{c}}$ & & & \\
\hline Urine & 4 & Neg. ${ }^{d}$ & Pos. ${ }^{e}$ & Neg. & Pos. & No & Yes & True Pos. \\
\hline Urine & 2 & Neg. & Pos. & Neg. & Neg. & No & No & False Neg. \\
\hline Fecal & 1 & Pos. & Pos. & Neg. & Neg. & Yes (5 days) & Yes & False Neg. \\
\hline Fecal & 1 & Pos. & Neg. & Pos. & - & Yes (1 mo $)$ & Yes & True Pos. \\
\hline Lymph node & 2 & Neg. & Pos. & Neg. & Pos. & No & No & True Pos. \\
\hline Lymph node & 1 & Neg. & Pos. & Neg. & Neg. & No & No & False Neg. \\
\hline Lymph node & 1 & Pos. & Neg. & Pos. & - & Yes $(7 \mathrm{mo})$ & Yes & True Pos. \\
\hline Lymph node & 1 & Pos. & Neg. & Pos. & - & Yes $(1 \mathrm{mo})$ & No & True Pos. \\
\hline Lymph node $f$ & 1 & Pos. & Neg. & Pos. & - & Yes (3 mo) & Yes & True Pos. \\
\hline Lymph node ${ }^{g}$ & 1 & Neg. & Neg. & Pos. & - & No & No & Inconclusive \\
\hline CSF & 3 & Neg. & Pos. & Neg. & Pos. & No & Yes & True Pos. \\
\hline Tissue biopsy & 2 & Pos. & Neg. & Pos. & - & Yes (4 mo) & Yes & True Pos. \\
\hline
\end{tabular}

${ }^{a}$ All patients had a clinical diagnosis of TB.

${ }^{b}$ For M. tuberculosis.

${ }^{c}$ Retested only the 13 specimens that were AMTDT negative and culture positive for M. tuberculosis. —, not retested.

${ }^{d}$ Neg., negative.

${ }^{e}$ Pos., positive.

${ }^{f}$ Specimens from three patients with active cervical tuberculous lymphadenitis, with compatible histology (granulomatous inflammation) and good response to TB treatment.

${ }^{g}$ From one patient whose histology showed granulomatous inflammation; response to TB treatment was good, and the patient probably had active cervical tuberculous lymphadenitis.

specimens the AMTDT has yielded overall sensitivities between 82 and $97 \%$ and specificities between 97 and 100\%. In contrast, there is considerably less experience with direct detection of $M$. tuberculosis in nonrespiratory specimens with the AMTDT $(13,25,30)$. Paradoxically, however, it is precisely extrapulmonary TB (tuberculous pleuritis, peritonitis, menin-

TABLE 3. Results of AMTDT and culture in nonrespiratory specimens

\begin{tabular}{lccr}
\hline \multirow{2}{*}{$\begin{array}{c}\text { Type of specimen and } \\
\text { AMTDT result }\end{array}$} & \multicolumn{2}{c}{ No. of cultures } & Total \\
\cline { 2 - 3 } & Positive & Negative & \\
\hline Urine & 9 & 0 & \\
Positive & $9^{a}$ & 22 & 31 \\
Negative & 18 & 22 & 40 \\
Total & & & \\
Fecal & 6 & $1^{b}$ & 7 \\
Positive & $9^{c}$ & 22 & 31 \\
Negative & 15 & 23 & 38 \\
Total & 14 & $4^{d}$ & \\
Lymph node & 3 & 7 & 18 \\
Positive & 17 & 11 & 10 \\
Negative & & & 28 \\
Total & 35 & 0 & 35 \\
Sterile fluid & $4^{e}$ & 47 & 51 \\
Positive & 39 & 47 & 86 \\
Negative & & & \\
Total & 7 & $2^{b}$ & 9 \\
Other & 0 & 23 & 23 \\
Positive & 7 & 25 & 32 \\
Negative & & & \\
Total & & &
\end{tabular}

${ }^{a}$ M. tuberculosis $(n=6)$ and M. gordonae $(n=3)$.

${ }^{b}$ TB treatment initiated for extrapulmonary disease.

${ }^{c}$ M. avium-M. intracellulare $(n=4)$, M. genavense $(n=3)$, M. xenopi $(n=1)$, and M. tuberculosis $(n=1)$.

${ }^{d}$ Three specimens from three patients with TB treatment initiated for active cervical tuberculous lymphadenitis; the other specimen corresponded to a patient who probably had active cervical tuberculous lymphadenitis (Table 2).

${ }^{e}$ M. tuberculosis $(n=3)$ and M. kansasii $(n=1)$. gitis, and lymph node TB, etc.) for which a rapid and accurate laboratory diagnosis is of prime importance. Recent evaluations of AMTDT performance have reported complete inhibition of the test when applied to a CSF specimen and a pleural aspirate specimen spiked with $M$. bovis BCG cells (12) and an unacceptable sensitivity of $20 \%$ for pleural exudates compared with culture on Löwenstein-Jensen medium (30). Very recently, however, the AMTDT was successfully adapted by Pfyffer et al. (25) for use with CSF and other nonrespiratory specimens. The need for proper sample preparation for amplification procedures to eliminate inhibitors that interfere with detection has been demonstrated in several studies $(1,3$, 13, 24-26).

The difficulties of using the AMTDT in detecting M. tuberculosis in nonrespiratory specimens, then, is well-known (12, $25,30)$. In the present study, two different SDS pretreatment procedures of such specimens, including some containing a high proportion of interfering compounds such as from urine, feces, fluids, and bone marrow aspirates, are described. Pretreatment with SDS-NaOH in 86 sterile body fluid samples (41 pleural exudates, 17 CSFs, 10 articular fluids, 17 ascitic fluids, and 1 pericardic fluid) yielded values for sensitivity, specificity, PPV, and NPV of 92.1, 100, 100, and 94.1\%, respectively, for the AMTDT. Pretreatment with SDS, involving extensive washing which is sufficient to remove any traces of detergent that might react with the assay, likewise proved successful in detecting specimens small loads of TB bacilli in bone marrow aspirates, although too few specimens were tested to permit statistical analysis (Table 1). Other authors $(25,26)$ have similarly reported success in pretreating samples with SDS, a detergent which denatures proteins and enzymes and eliminates most of the inhibitory compounds present in body fluids. As for nonrespiratory specimens other than body fluids and bone marrow aspirates, we found that pretreatment with SDS is also mandatory to obtain consistent and reproducible AMTDT results. The sensitivity and specificity of AMTDT of 85.7 and $100 \%$, respectively, obtained after SDS treatment, were acceptable for detection of $M$. tuberculosis in all our pretreated 
samples; these results were similar to those of Pfyffer et al. (25), who achieved a sensitivity and specificity of 93.1 and $97.7 \%$, respectively, in specimens pretreated with SDS-NaOH, in contrast with those of Vlaspolder et al. (30), who reported a sensitivity of only $20 \%$ in pleural exudates pretreated with $\mathrm{N}$-acetyl-L-cysteine- $\mathrm{NaOH}$.

The 13 false-negative AMTDT results that we report (six urine samples, one fecal sample, three lymph nodes, and three CSF samples) (Table 2) illustrated another sampling problem commonly encountered with small loads of TB bacilli in nonrespiratory specimens as a result of the tendency of the bacilli to clump together. As previously described $(4,25)$, increasing the initial amount of specimen from 50 to $500 \mu l$ and subjecting the specimen to subsequent centrifugation provides the AMTDT with a larger target. Our data show clearly that screening 500 instead $50 \mu \mathrm{l}$ of sediment improves the sensitivity of the test ( 85.7 and $95.6 \%$ in 50 - and $500-\mu$ l nonrespiratory specimens, respectively) without adversely affecting specificity.

We conclude that because the AMTDT is able to detect stable rRNA from noncultivable bacilli in pretreated nonrespiratory specimens, the test is not useful for monitoring patients receiving treatment. However, before this can be considered, additional studies need to be realized to corroborate the existing data in patients undergoing antimicrobial therapy. Published reports $(18,25,26,30)$ clearly show that a patient receiving such treatment can remain AMTDT positive after cultures become negative, possibly because $16 \mathrm{~S}$ rRNA molecules are protected after cell death by ribosomal proteins (29). Despite this handicap, we believe that the AMTDT can be used in diagnosis of partially treated patients arriving with inconclusive medical data that are often culture negative and may be acid-fast staining positive or negative. Our findings underscore the need to design a faster, more sensitive direct test to replace acid-fast staining and culture so that patients in treatment can be monitored adequately.

\section{REFERENCES}

1. Abe, C., K. Hirano, M. Wada, Y. Kazumi, M. Takahashi, Y. Fukasawa, T. Yoshimura, C. Miyagi, and S. Goto. 1993. Detection of Mycobacterium tuberculosis in clinical specimens by polymerase chain reaction and Gen-Probe Amplified Mycobacterium Tuberculosis Direct Test. J. Clin. Microbiol. 31: 3270-3274.

2. Abe, C., S. Hosojima, Y. Fucosava, Y. Kazumi, M. Takahashi, K. Hirao, and T. Mori. 1992. Comparison of MB-Check, BACTEC, and egg-based media for recovery of mycobacteria. J. Clin. Microbiol. 30:878-881.

3. Bodmer, T., A. Gurtner, K. Schopfer, and L. Matter. 1994. Screening of respiratory tract specimens for the presence of Mycobacterium tuberculosis by using the Gen-Probe Amplified Mycobacterium Tuberculosis Direct Test. J. Clin. Microbiol. 32:1483-1487.

4. Bodmer, T., E. Möckl, K. Mühlemann, and L. Matter. 1996. Improved performance of Gen-Probe Amplified Mycobacterium Tuberculosis Direct Test when 500 instead of 50 microliters of decontaminated sediment is used. J. Clin. Microbiol. 34:222-223.

5. Brisson-Noël, A., C. Aznar, C. Chureau, S. Nguyen, C. Pierre, M. Bartoli, R. Bonete, G. Pialoux, B. Gicquel, and G. Garrigue. 1991. Diagnosis of tuberculosis by amplification of mycobacterial DNA in clinical practical evaluation. Lancet 338:364-366.

6. Butler, W. R., and J. O. Kilburn. 1988. Identification of major slowly growing pathogenic mycobacteria and Mycobacterium gordonae by high-performance liquid chromatography of their mycolic acids. J. Clin. Microbiol. 26:50-53.

7. Chain, K. 1995. Clinical microscopy, p. 33-51. In P. R. Murray, E. J. Baron, M. A. Pfaller, F. C. Tenover, and R. H. Yolken (ed.), Manual of clinical microbiology, 6th ed. American Society for Microbiology, Washington, D.C.

8. Clarridge, J. E., III, R. M. Shawar, T. M. Shinninck, and B. B. Plicaytis. 1993. Large-scale use of polymerase chain reaction for detection of Mycobacterium tuberculosis in a routine mycobacteriology laboratory. J. Clin. Microbiol. 31:2049-2056.

9. Coletsos, P. J. 1960. Milieu et modalités de culture adaptés a la réanimation et à la multiplication in vitro de $M$. tuberculosis de vitalité réduite de viabilité éphemère ou en état de quiescence. Ann. Inst. Pasteur 99:457-495.

10. Daniel, T. M. 1990. The rapid diagnosis of tuberculosis: a selective review. J. Lab. Clin. Med. 116:277-282.

11. Del Portillo, P., L. A. Murillo, and M. E. Patarroyo. 1991. Amplification of a species-specific DNA fragment of Mycobacterium tuberculosis and its possible use in diagnosis. J. Clin. Microbiol. 29:2163-2168.

12. Ehlers, S., M. Pirmann, W. Zaki, and H. Halin. 1994. Evaluation of a commercial rRNA target amplification assay for detection of Mycobacterium tuberculosis complex in respiratory specimens. Eur. J. Clin. Microbiol. Infect. Dis. 13:827-829.

13. Ehlers, S., R. Ignatius, T. Regnath, and H. Hahn. 1996. Diagnosis of extrapulmonary tuberculosis by Gen-Probe Amplified Mycobacterium tuberculosis Direct Test. J. Clin. Microbiol. 34:2275-2279.

14. Evans, K. D., A. S. Nakasone, P. A. Sutherland, L. M. de la Maza, and E. M. Peterson. 1992. Identification of Mycobacterium tuberculosis and Mycobacterium avium- $M$. intracellulare directly from primary BACTEC cultures by using acridinium-ester-labeled DNA probes. J. Clin. Microbiol. 30:24272431.

15. Forbes, B. A., and K. E. S. Hicks. 1993. Direct detection of Mycobacterium tuberculosis in respiratory specimens in a clinical laboratory by polymerase chain reaction. J. Clin. Microbiol. 31:1688-1694.

16. Heifets, L. B., and R. C. Good. 1994. Current laboratory methods for the diagnosis of tuberculosis, p. 85-110. In B. R. Bloom (ed.), Tuberculosis: pathogenesis, protection, and control. American Society for Microbiology, Washington, D.C.

17. Hermans, P. W. M., A. R. J. Schuitema, D. Van Soolingen, C. P. H. J. Verstynen, E. M. Bik, J. E. R. Thole, A. H. J. Kolk, and J. D. A. van Embden. 1990. Specific detection of Mycobacterium tuberculosis complex strains by polymerase chain reaction. J. Clin. Microbiol. 28:1204-1213.

18. Jonas, V., M. J. Alden, J. I. Curry, K. Kamisango, C. A. Knott, R. Lankford, J. M. Wolfe, and D. F. Moore. 1993. Detection and identification of Mycobacterium tuberculosis directly from sputum sediments by amplification of rRNA. J. Clin. Microbiol. 31:2410-2416.

19. Kox, L. F. F., D. Rhienthong, A. Medo Miranda, N. Udomsantisuk, K. Ellis J. van Leeuwen, S. van Heusden, S. Kuijper, and A. H. J. Kolk. 1994. A more reliable PCR for detection of Mycobacterium tuberculosis in clinical samples. J. Clin. Microbiol. 32:672-678.

20. La Rocco, M. T., A. Wagner, H. Ocera, and E. Macias. 1994. Evaluation of a commercial rRNA amplification assay for direct detection of Mycobacterium tuberculosis. Eur. J. Clin. Microbiol. Infect. Dis. 33:726-731.

21. Luquin, M., V. Ausina, F. López-Calahorra, F. Belda, M. García-Barceló, C Celma, and G. Prats. 1991. Evaluation of practical chromatographic procedures for identification of clinical isolates of mycobacteria. J. Clin. Microbiol. 29:120-130.

22. Miller, N., S. G. Hernandez, and T. J. Cleary. 1994. Evaluation of Gen-Probe Amplified Mycobacterium Tuberculosis Direct Test and PCR for detection of Mycobacterium tuberculosis in clinical specimens. J. Clin. Microbiol. 32: 393-397.

23. Noordhoek, G. T., A. H. J. Kolk, G. Bjune, D. Catty, J. W. Dale, P. E. M. Fine, P. Godfrey-Faussett, S.-N. Cho, T. Shinnick, S. B. Svenson, S. Wilson, and J. D. A. van Embden. 1994. Sensitivity and specificity of PCR for detection of Mycobacterium tuberculosis: a blind comparison study among seven laboratories. J. Clin. Microbiol. 32:277-284.

24. Pfyffer, G. E. 1994. Amplification techniques: hope or illusion in the direct detection of tuberculosis. Med. Microbiol. Lett. 3:335-347.

25. Pfyffer, G. E., P. Kissling, E. M. I. Jahn, H.-M. Welscher, M. Salfinger, and R. Weber. 1996. Diagnostic performance of Amplified Mycobacterium tuberculosis Direct Test with cerebrospinal fluid, other nonrespiratory, and respiratory specimens. J. Clin. Microbiol. 34:834-841.

26. Pfyffer, G. E., P. Kissling, R. Wirth, and R. Weber. 1994. Direct detection of Mycobacterium tuberculosis complex in respiratory specimens by a targetamplified test system. J. Clin. Microbiol. 32:918-923.

27. Reisner, B. S., A. M. Gatson, and G. L. Woods. 1994. Use of Gen-Probe AccuProbes to identify Mycobacterium avium complex, Mycobacterium tuberculosis complex, Mycobacterium kansasii, and Mycobacterium gordonae directly from BACTEC TB broth cultures. J. Clin. Microbiol. 32:2995-2998.

28. Salfinger, M., and F. M. Kafader. 1987. Comparison of two pretreatment methods for the detection of mycobacteria of BACTEC and LöwensteinJensen slants. J. Microbiol. Methods 6:315-321.

29. van der Vliet, G. M. E., P. Schepers, R. A. F. Schukkink, B. van Gemen, and P. R. Klatser. 1994. Assessment of mycobacterial viability by RNA amplification. Antimicrob. Agents Chemother. 38:1959-1965.

30. Vlaspolder, F., P. Singer, and C. Roggeveen. 1995. Diagnostic value of an amplification method (Gen-Probe) compared with that of culture for diagnosis of tuberculosis. J. Clin. Microbiol. 33:2699-2703. 